\title{
Concerns about Validity of Caesarian Section for Foetal Distress with No Risk in Mother - Baby
}

\author{
Chhabra S* and Mandar K \\ Department of Obstetrics Gynaecology, Mahatma Gandhi \\ Institute of Medical Sciences, Maharashtra, India \\ *Corresponding author: Chhabra S, Department of \\ Obstetrics Gynaecology, Mahatma Gandhi Institute of \\ Medical Sciences, Kasturba Health Society, Sevagram, \\ Wardha, Maharashtra, India
}

Received: J une 20, 2017; Accepted: August 08, 2017; Published: August 16, 2017

\begin{abstract}
Background: Diagnosis of Foetal Distress (FD) is nonspecific, because of low positive predictive value. Many times after interventions, infant is in good condition at birth with normal Apgar score or umbilical cord blood $\mathrm{pH}$ or both. Equally true is that some babies are limp, sometimes still born at Cesarean Birth (CB) for FD. It is essential to understand modalities of authentic, timely diagnosis of FD, causes of true FD in women without risk.
\end{abstract}

Aim: Study was done to know frequency of diagnosis of intrapartum FD and appropriateness of caesarian section in cases with no risk factors.

Methods: Analytical study of 252 women admitted to labour area with term gestation, vertex presentation, no apparent risk for FD, were enrolled. Diagnosis of FD was made by intrapartum intermittent foetal heart auscultation (IFHA) and/ or Non-Stress Test (NST) and/or intrapartum presence of meconium in liquor amnii.

Results: There was no still birth, 211 (83.74\%) babies were vigorous at birth after C Birth for FD, 41(16.26\%) were admitted to Neonatal Intensive Unit (NICU), $40(97.56 \%)$ recovered completely with one neonatal death, $(2.43 \%$ of NICU admissions, $0.39 \%$ of $252 \mathrm{CS}$ ) due to severe birth asphyxia because of congenital heart disease in baby, missed during pregnancy. CS for unexplained FD accounted for $2.74 \%$ of births (9186), $7.44 \%$ of CS (3385). Apgar scores had poor correlation with perinatal asphyxia, umbilical cord blood $\mathrm{pH}$.

Conclusion: In around $66 \%$ women CS for FD without any risk factor, there was no abnormality, even during CS. Babies were vigorous at birth, obviously, over diagnosis of FD, through conventional diagnostic modalities. Research is needed for authentic diagnostics for non- reassuring fetal status, non conventional or unknown risk factors, responsible for fetal asphyxia which leads to diagnosis of FD.

Keywords: Caesarean Section; Fetal Distress; Validity; Risk Factors

\section{Introduction}

\section{Background}

Years back, Parer and Livingston [1] reported that the lack of clear definition of Fetal Distress (FD) compounded the difficulty in making an accurate diagnosis and initiation of appropriate treatment. The situation continues to be the same. ACOG [2] in its guidelines described the term FD as imprecise, which resulted in inappropriate action, such as an unnecessary urgent delivery under general anaesthesia. Diagnosis of FD is nonspecific because of low positive predictive value even in high-risk populations. Many a times after interventions, the infant is in good condition at birth, with normal Apgar score or umbilical cord blood $\mathrm{pH}$ or both. Equally true is the fact that some babies are limp, sometimes still born at Cesarean Birth (CB) for FD. It is essential to understand the modalities for authentic timely diagnosis of FD and causes of true FD without risk factors in mothers and/or baby.

\section{Objectives}

Present study was done to know the frequency of diagnosis of intrapartum FD, appropriateness of Caesarean Section (CS) performed for FD in cases with no risk factors, during pregnancy or labour and causes missed during pregnancy as well as at onset of labour but evident during birth.

\section{Materials and Methods}

The present prospective study was carried out over 2 years at a rural referral institute. Approval of the ethics committee of the institute, and informed consent from the study subjects were taken, for enrolment of the mother, foetus and the newborn in the study. It was an observational analytical study of women admitted to the labour area with term gestation with no apparent risk factor for FD. There were 9186 births during the study period, 5801 (63.15\%) vaginal \& 3385 (36.85\%) by CS. Of the 3385 CS, 2370 (70.01\% of all CS) were emergency CS and 948 (40\% of all emergency CS) were for FD, $73.42 \%$ (696 of 948) had some or other risk factor diagnosed during pregnancy or at the time of admission to labour area and the remaining 252 (26.58\% of CS for FD) women had no risk factor. So CS for unexplained FD accounted for $2.74 \%$ of all births (9186) and $7.44 \%$ of all CS (3385). These 252 women were admitted to the labour area with term, singleton pregnancy with vertex presentation. They were
Austin J Obstet Gynecol - Volume 4 Issue 2 - 2017

Submit your Manuscript | www.austinpublishinggroup.com

Chhabra et al. () All rights are reserved
Citation: Chhabra S and Mandar K. Concerns about Validity of Caesarian Section for Foetal Distress with No Risk in Mother - Baby. Austin J Obstet Gynecol. 2017; 4(2): 1072. 
Table 1: Meconium and Intermittent Fetal Heart Rate and NST.

\begin{tabular}{|c|c|c|c|c|c|c|c|c|}
\hline \multirow{2}{*}{ Intrapartum liquor } & \multirow{2}{*}{ Number of cases } & \multirow{2}{*}{$\%$} & \multicolumn{3}{|c|}{ Intermittent Auscultation } & \multicolumn{3}{|c|}{ NST } \\
\hline & & & Tachycardia & Bradycardia & Normal & Cat - I & Cat - II & Cat - III \\
\hline Thick & 53 & 21 & 6 & 5 & 42 & 10 & 18 & 46 \\
\hline Thin & 47 & 18.7 & 3 & 5 & 39 & 121 & 2 & 33 \\
\hline Clear & 151 & 59.9 & 26 & 14 & 111 & 33 & 13 & 105 \\
\hline Blood & 1 & 0.4 & - & - & 1 & 1 & 0 & 0 \\
\hline \multirow{2}{*}{ Total } & \multirow{2}{*}{252} & \multirow{2}{*}{100} & 35 & 24 & 193 & 165 & 33 & 184 \\
\hline & & & \multicolumn{3}{|c|}{252} & \multicolumn{3}{|c|}{252} \\
\hline
\end{tabular}

Table 2: Summary of intraoperative findings, \& risk factor, not detected earlier.

\begin{tabular}{|c|c|c|c|c|c|c|c|c|c|}
\hline \multirow{2}{*}{ Sr. No. } & \multirow{2}{*}{ Intraoperative Findings } & \multirow{2}{*}{ Number of Cases } & \multirow{2}{*}{$\%$} & \multicolumn{3}{|c|}{ Intermittent Auscultation } & \multicolumn{3}{|c|}{ NST } \\
\hline & & & & Tachycardia & Bradycardia & Normal & Cat -1 & Cat - II & Cat-III \\
\hline 1 & Thick meconium in liquor amnii & 53 & 21 & 6 & 5 & 42 & 10 & 2 & 41 \\
\hline 2 & Blood stained liquor amniii & 1 & 0.4 & & & 1 & 1 & & \\
\hline 3 & Retroplacental clots & 2 & 0.79 & 1 & & 1 & 1 & & 1 \\
\hline 4 & Cord around neck & 27 & 10.7 & 4 & 2 & 21 & 4 & 2 & 21 \\
\hline 5 & Heart disease in baby & 1 & 0.4 & & & 1 & & 1 & \\
\hline & Total & 84 & 33.3 & 11 & 7 & 66 & 16 & 5 & 63 \\
\hline & Normal intraoperative & 168 & 66.7 & 24 & 17 & 127 & 149 & 28 & 121 \\
\hline
\end{tabular}

monitored with intrapartum Intermittent Foetal Heart Auscultation (IFHA), Non-Stress Test (NST), and for the presence of meconium in liquor amni. Women who were diagnosed as intrapartum FD and intervened by CS were the study subjects. Diagnosis of FD was made by IFHA and/or NST, \& /or intrapartum presence of thick meconium in liquor amni. A tool was made for recording the details of the cases. No separate protocol was provided to clinicians but similar modalities of monitoring and recording were adopted in all the cases. Detailed information at admission to labour area, demographic profile, physical, obstetric examination, NST, IFHA \& meconium in liquor amnii were recorded. NST results were categorized as reactive or nonreactive according to recent ACOG guideline [3]. IFHR was done in all the cases with hand held digital Doppler, half hourly in active first stage \& every 5 minutes during second stage of labour, in accordance with joint AAP \& ACOG guidelines [4]. FHR was interpreted as mild Tachycardia $>160$, Severe Tachycardia $>180$, mild bradycardia $<110$, moderate bradycardia $<100$ and $<80 \mathrm{bpm}$ severe diagnosed as FD. CS for FD was performed for persisting bradycardia or persisting tachycardia. During CS, the intraoperative details, meconium in liquor amnii, abnormalities of placenta \& umbilical vessels, details of condition of the newborn at birth. Apgar scores at $1,5,10$ minutes and resuscitative measures needed for the baby were recorded. Umbilical cord blood $\mathrm{PH}$ immediately after the delivery of the neonate was done. $\mathrm{PH}$ of 7.2-7.36 was considered normal, 7.2 to 7.0 , as moderate and $<7.0$ as severe acidosis. Neonatal Intensive Care Unit (NICU) admission \& interventions if any were recorded. Postpartum follow up of newborn was done for 7 days (Table 1).

\section{Results}

Mean age of the women enrolled into study was $24.4 \pm 3.38$ years, 198 (78.57\%) primigravida, 47 (18.65\%) second, third gravida and 7 $(2.78 \%)$ were fourth gravida. Primigravida $78 \%$ significantly more than overall $48 \%$ during the same period. The mean gestational age was $38.74 \pm 1.06$. One hundred and eighty two $(72.23 \%)$ women were admitted in latent phase of labour, 46 (18.25\%) in early active phase of labour, 24 (9.52\%) late active phase. Of the 252 cases who underwent CS for FD, 56 (22.22\%) had category I (reassuring), 17 (6.75\%) category II (indeterminate) and 179 (71.03\%) had category III (nonassuring) pattern of NST. IFHA, which lead to diagnosis of FD and CS, recorded persisting tachycardia in $35(13.89 \%)$ cases, mild in $14(5.55 \%)$ \& severe in $21(8.33 \%), 24(9.52 \%)$ persistent bradycardia, (9 (3.57\%) mild, 14 (5.54\%) moderate \& one (0.39\%) severe bradycardia). Of the 252 CS for FD, 140 (55.57\%) cases had FHR changes on IFHA as well as NST and $86(34.12 \%)$ had moderate to thick meconium in liquor amnii and $26(10.31 \%)$ had abnormalities in all the three (NST, IFHA, as well as moderate or thick meconium in liquor). Finally after CS, 33 (13\%) had moderate meconium, 53 (21.03\%) had thick meconium, $14(5.5 \%)$ thin meconium, one (0.39\%) had blood stained liquor \& in 151 (59.92\%) cases, the liquor was absolutely clear. Of the 53 cases who had thick meconium, 6 (11.3\%) had foetal tachycardia, 5 (9.4\%) bradycardia \& in $42(79.3 \%)$ cases foetal heart was normal. NST was Category - III in 46 (86.79\%), category - II, in 18 (33.96\%) \& category - I in 10 (18.86\%). On IFHA severe bradycardia or persisting tachycardia were present in $38(15.07 \%)$ cases with clear or thin meconium in liquor. In 138(54.76\%) cases with category - III NST, liquor amni was clear or with thin meconium.

There was no stillbirth. 211 (83.74\%) babies were vigorous at birth after CS for FD and 41 (16.26\%) were admitted to NICU (Apgar score $<7$ at $1 \mathrm{~min})$ with recovery in $40(97.56 \%)$ babies. Of the 40 babies who had recovery, 5 (12.5\%) had three or less Apgar \& remaining 35 (87.5\%) had Apgar scores between 4 to 6 at $1 \mathrm{~min}$. Apgar scores had poor correlation with foetal hypoxia or perinatal asphyxia, with the value of umbilical cord blood ph, a direct measure of acidosis. There was one neonatal death $(2.43 \%$ of NICU admissions, $0.39 \%$ 
Table 3: Intermittent Auscultation, Sensitivity \& Specificity.

\begin{tabular}{|c|c|c|c|c|c|}
\hline & \multicolumn{2}{|c|}{ Degree \& presence or absence of Birth Asphyxia } & \\
\hline $\begin{array}{c}\text { Intermittent } \\
\text { Auscultation }\end{array}$ & $\begin{array}{c}\text { Severe } \\
\text { Acidosis }\end{array}$ & $\begin{array}{c}\text { Moderate } \\
\text { Acidosis }\end{array}$ & $\begin{array}{c}\text { Birth } \\
\text { Asphyxia* }\end{array}$ & Normal & Total \\
\hline Bradycardia & 4 & 15 & 19 & 5 & 24 \\
\hline Tachycardia & 2 & 25 & 27 & 8 & 35 \\
\hline Abnormal FHR & 6 & 40 & 46 & 13 & 59 \\
\hline Normal FHR & 0 & 142 & 142 & $\mathbf{5 1}$ & 193 \\
\hline TOTAL & 6 & 182 & 188 & 64 & 252 \\
\hline
\end{tabular}

*Moderate \& severe acidosis are clubbed into birth asphyxia cases.

** Similarly, tachycardia \& bradycardia, both representing abnormal FHR pattern clubbed together.

Table 4: NST / Umbilical Cord Blood pH.

\begin{tabular}{|c|c|c|c|c|c|}
\hline & \multicolumn{3}{|c|}{ Degree \& Presence or Absence of Birth } & \\
\hline NST / EFMyxia & $\begin{array}{c}\text { Asphy } \\
\text { Acidosis }\end{array}$ & $\begin{array}{c}\text { Moderate } \\
\text { Acidosis }\end{array}$ & BA & Normal & Total \\
\hline $\begin{array}{c}\text { Category - III (Non- } \\
\text { reassuring) }\end{array}$ & 6 & 126 & 132 & 47 & 179 \\
\hline $\begin{array}{c}\text { Category - II } \\
\text { (Indeterminate) }\end{array}$ & 0 & 13 & 13 & 4 & 17 \\
\hline $\begin{array}{c}\text { Category - I } \\
\text { (Reassuring) }\end{array}$ & 0 & 43 & 43 & 13 & $\mathbf{5 6}$ \\
\hline TOTAL & 6 & 182 & 188 & 64 & 252 \\
\hline
\end{tabular}

BA- Birth asphyxia.

of 252 cases studied) of the baby with Apgar score one with severe birth asphyxia because of congenital heart disease, missed during pregnancy.

Overall 66 (26.19\%) babies had umbilical blood gas $\mathrm{pH}$ of 7.2-7 overall $36,182(72.22 \%)$ had $\mathrm{pH}$ of 7.2 to 7.0 with moderate acidosis and in $4(1.58 \%)$, there was severe acidosis $<7.0$. with birth asphyxia.

During CB, cord around neck was detected in 27 babies (10.71\%), placental abruption with retro-placental clot in $2(0.79 \%)$, heart disease in one baby. In 53 (21.03\%) liquor amini had thick meconium \& liquor was blood stained in one $(0.39 \%)$ not diagnosed during decision of CS. In 168 (66.66\%) cases there was no abnormality neither in placenta, nor cord or baby during CS or immediate postpartum. Of the $53(21.03 \%)$ cases in whom intrapartum thick meconium was detected, there was persisting foetal tachycardia in $6(11.3 \%)$, bradycardia in $5(9.4 \%)$ and $42(79.3 \%)$ had normal FHR, NST was of category - III in 41 (77.35\%), category II in $2(3.77 \%) \& 10(18.86 \%)$ had category - I NST. In the case with intra operative detection of abruption and blood in liquor, IFHR was normal \& NST was category I. Of the $2(2.4 \%)$ cases with CS for persisting tachycardia, in one Table 5: Meconium and Cord Blood pH.
(1.2\%) NST was category -III, \& one each category - I. In 27 (10.71\%) cases in whom cord around the neck was detected during CS, NST was category - III normal IFHR in 21 (77.77\%), 4 (14.81\%) had category - I NST and tachycardia \& $2(7.40 \%)$ had category - II NST and bradycardia (Table 2).

Persisting fetal tachycardia on IHFA lead to CB for FD in 35 (13.88\%) cases, 14 (5.55\%) (Persisting mild tachycardia (>160bpm), $21(8.33 \%)$ severe tachycardia $(>180 \mathrm{bpm})$. Two out of 21 with severe tachycardia were admitted to NICU. The mean Apgar score was 6 and mean umbilical cord blood $\mathrm{pH}$ was 7.18. All babies who had CB for persisting mild tachycardia were vigorous at birth with mean Apgar score of 7 , mean umbilical cord blood $\mathrm{pH}$ of 7.18. FD was diagnosed because of persisting foetal bradycardia in 22 (8.73\%), (13 (5.15\%) moderate bradycardia ( $<100 \mathrm{bpm}), 5$ of these 13 babies were admitted to NICU, mean Apgar score was 5 \& mean umbilical cord blood gas ph 7 . There was persisting mild bradycardia $(<110 \mathrm{bpm})$ in $8(3.17 \%)$ cases, babies were vigorous at birth with mean Apgar score 7 umbilical cord blood $\mathrm{pH}$ of 7 . Severe foetal bradycardia $(<80 \mathrm{bpm})$ lead to CB in one $(0.39 \%)$ case. Baby had Apgar score 1 at $1 \mathrm{~min} \&$ umbilical cord blood $\mathrm{pH}$ was 6.9 and baby was admitted to NICU. Finally meconium was present in $100(39.68 \%)$ cases with babies mean Apgar score 7 \& mean umbilical artery cord blood pH 7.18 (Table 3).

IFHA for diagnosis of FD had sensitivity of $24.47 \%$ (95\% CI: $18.51 \%$ to $31.26 \%$ ), the specificity $79.69 \%$ (95\% CI: $67.77 \%$ to $88.71 \%$ ). Sensitivity of NST was $75.43 \%$ (95\% CI: $68.36 \%$ to $81.61 \%$ ) and specificity: $21.67 \%$ (95\% CI: $12.08 \%$ to $34.20 \%$ ) (Table 4 ). Meconium in liquor amni had specificity, sensitivity of $22.87 \%$ (95\% CI: $17.08 \%$ to $29.55 \%$ ) \& $82.81 \%$ (95\% CI: $71.32 \%$ to $91.08 \%$ ) respectively. The analysis revealed that of $100(60.31 \%)$ cases of CS for unexplained FD which had meconium, of 53 cases with thick meconium 11 (20.75\%) babies really had asphyxia \& 42( 79.3 ) were vigorous at birth (Table 5).

During CS, 168(66.66\%) women had no abnormality, 27 (16.17\%) of them needed NICU management. So overall 140 (55\%) cases of all $\mathrm{CS}$ for $\mathrm{FD}$, did not have any abnormality during pregnancy, labour and CS did not need any special care (1.83\% of all CS, $2.64 \%$ of all emergency CS, $1.52 \%$ of all births), CS could have been avoided. In eighty four babies there were intraoperative abnormalities, but only $14(5.5 \%)$ of them needed NICU admission and 71 babies were normal at birth (Table 6).

\section{Discussion}

FD, term used in day to day clinical scenario, is deemed

\begin{tabular}{|c|c|c|c|c|c|c|c|}
\hline \multirow{3}{*}{ Intrapartum colour of liquor } & \multirow{3}{*}{ Number of cases } & \multirow{3}{*}{$\%$} & \multicolumn{3}{|c|}{ Birth Asphyxia } & \multirow{3}{*}{ Normal } & \multirow{3}{*}{ Total } \\
\hline & & & \multirow{2}{*}{ Severe Acidosis } & Moderate & \multirow{2}{*}{ BA } & & \\
\hline & & & & Acidosis & & & \\
\hline Thick & 53 & 21 & 2 & 40 & 42 & 11 & 53 \\
\hline Blood & 1 & 0.4 & 0 & 1 & 1 & 0 & 1 \\
\hline \multicolumn{3}{|c|}{ Clinically significant liquor types } & 2 & 41 & 43 (17.06\%) & $11(4.36 \%)$ & 54 \\
\hline Clear & 151 & 59.9 & 3 & 107 & 110 & 41 & 151 \\
\hline Thin & 47 & 18.7 & 1 & 34 & 35 & 12 & 47 \\
\hline \multicolumn{3}{|c|}{ Normal Liquor } & 4 & 141 & $145(57.53 \%)$ & $53(21.03 \%)$ & 198 \\
\hline Total & 252 & 100 & 6 & 202 & 208 & 44 & 252 \\
\hline
\end{tabular}


Table 6: NST, IFHR Apgar score, NICU Admission.

\begin{tabular}{|c|c|c|c|c|c|c|c|}
\hline \multicolumn{3}{|c|}{ Method of diagnosing foetal distress } & No of Cases & $\%$ & $\begin{array}{l}\text { Mean Apgar } \\
\text { At } 1 \text { min }\end{array}$ & Mean Umbilical Cord Blood pH & NICU Admissions \\
\hline \multirow{8}{*}{ EFM/NST } & \multirow{4}{*}{ Category -III } & Early & 29 & 11.5 & 8 & 7.14 & 5 \\
\hline & & Late & 11 & 4.37 & 5.29 & 7.11 & 5 \\
\hline & & Variable & 71 & 28.6 & 6.3 & 7.17 & 11 \\
\hline & & Loss of variability & 68 & 27 & 8.26 & 7.13 & 12 \\
\hline & \multicolumn{2}{|c|}{ Category - III Non-Reassuring (Total) } & 179 & 71 & - & - & - \\
\hline & \multicolumn{2}{|c|}{ Category - II (Indeterminate) } & 17 & 6.74 & 8 & 7.22 & - \\
\hline & \multicolumn{2}{|c|}{ Category - I (Reassuring) } & 56 & 22.2 & 8 & 7.28 & - \\
\hline & \multicolumn{2}{|c|}{ Total EFM/NST } & 252 & 100 & - & - & - \\
\hline \multirow{7}{*}{ Intermittent Auscultation } & \multirow{3}{*}{ Bradycardia } & Mild (<110bpm) & 9 & 3.57 & 6 & 7 & - \\
\hline & & Moderate $(<100 \mathrm{bpm})$ & 14 & 5.54 & 5 & 7 & 5 \\
\hline & & Severe $(<80 \mathrm{bpm})$ & 1 & 0.39 & 2.75 & 6.9 & 1 \\
\hline & \multirow{2}{*}{ Tachycardia } & Mild (>160bpm) & 14 & 5.55 & 6 & 7.18 & - \\
\hline & & Severe (>180bpm) & 21 & 8.33 & 6 & 7.18 & 2 \\
\hline & \multicolumn{2}{|c|}{ Normal (110 - 160bpm) } & 193 & 76.6 & 8 & 7.28 & - \\
\hline & \multicolumn{2}{|c|}{ Total Intermittent Auscultation } & 252 & 100 & - & - & - \\
\hline \multicolumn{3}{|c|}{ Total Number of cases diagnosed on FHR basis } & 153 & 60.7 & - & - & - \\
\hline \multicolumn{3}{|c|}{ Meconium staining of amniotic fluid } & 99 & 39.3 & 7 & 7.15 & - \\
\hline
\end{tabular}

inappropriate by many, since it just means clinician's interpretation of fetal status, and denotes that the clinician is not satisfied with the fetal status. Major drawback with the term FD is that it does not convey the cause or severity or implications on the outcome. Validity of interventions with this diagnosis is known only after the intervention has been done, too late. Women may be having some disorder/risk diagnosed during pregnancy which could affect the fetal cardio respiratory status and cause FD. But some women with no risk factor at all are diagnosed with FD and intervened by CS. Baby is vigorous at birth and beyond. So it was decided to look into some of the issues.

Substantial number, 252 (26.58\% of all CS for FD, 948) were performed for FD without any obvious risk factors, in the mother or the baby during pregnancy or at the onset of labour. However of the $252 \mathrm{CS}$, in 30 (12\%) abnormalities were detected intraoperatively, not known prior to CS. Additional 3 (1.20\%) had concealed placental abruption detected during CS. Of the 252 cases, overall 56 (22.22\%) had category I, 17 (6.75\%) category II and 179 (71.03\%) had category III NST. Most had variable deceleration [71 (28.57\%)], loss of foetal heart rate variability was in $69(26.99 \%)$ cases, early decelerations in $29(11.51 \%)$ cases and late decelerations in $11(4.37 \%)$ cases. In 27 (10.71\%) cases there was cord around the neck of the baby \& one (0.40\%) baby had cardiac anomaly.

Of the 252 women in 167 (66.26\%) cases, no abnormality was detected even during CS. Of these 167 cases, 140 (63\%) babies were vigorous at birth, but 27 (16.17\%) needed NICU management. So around $66 \%$ women who had CS for FD did not have any abnormality, even during CS. obviously over diagnosis of FD, through conventional modalities of diagnosis. Al-Abid [5] and Odongo [6] have also reported such findings. A study done by Low [7] of term pregnancies with diagnosis of foetal asphyxia revealed that in $63 \%$ cases no known risk factors for foetal asphyxia were present.
Foetal asphyxia/FD can occur in cases without any obvious risk factor, making it essential to conduct studies regarding the causes of fetal asphyxia and also authenticity of diagnosis of FD. This is essential because women with disorders which predispose to foetal asphyxia should be managed appropriately and timely and those without such disorders should be managed in such a way that unnecessary interventions are not done. ACOG [2] and Rosser [8] also reported that an important aspect of foetal monitoring is the high or false positive rate of FD associated with most IPFHR monitoring methods, coupled with the poor ability to interpret monitoring results that may both contribute to unnecessary interventions. Nelson et al [9] reported that despite the rise in CS rate to excess of $25 \%$,neither the rate of Cerebral Palsy (CP), nor that of any other childhood neurologic problems have been affected in the slightest, weighing against the inappropriate \& alarming rise in CS rate due to false-positive rate of $99.8 \%$ in EFM. Unfortunately, precise information about the frequency of false-positive results is lacking, and this lack is due in large part to the absence of accepted definition of FD [10]. IFHA criticized in all low risk cases with advent of EFM long back was reanalyzed and has been advised as standard of care by joint committee of American Association of Paediatricians [4]. The suggested duration \& frequency of IA is minimum of 60 seconds, at least every 30 minutes after a contraction in the active stage and every 5 minutes in the second stage of labour [3]. In the presence of increased risk of perinatal death, CP or neonatal encephalopathy or with oxytocin for induction or augmentation, continuous EFM has been suggested [11].

From time to time several hospital based studies have proved that various antepartum, intrapartum maternal \& fetal or postnatal risk factors are responsible for perinatal asphyxia. Chauhan et al [12] conducted a meta-analysis comprising of 169 articles, and 37 reports and concluded that the overall risk of prompt CB for foetal concern 
was $3.1 \%$. In the present study it was $2.74 \%$. Any mother, if screened and found to be with no risk factor has chance of being diagnosed as FD either due to intrapartum decreased oxygenation of foetus because of suboptimal uterine perfusion, placental dysfunction, and other intrapartum events or with false alarm. The research needs to continue.

Present study revealed better sensitivity (75.43\%), but low specificity $(21.67 \%)$ with NST in predicting perinatal asphyxia, high false positivity in detecting foetal status diagnosed as FD in women without risk factors, thus increasing the risk of CS without actual need. After Cochrane Database Systematic review Devane et al [13] reported that women who were low risk at admission for labour, should have been informed that admission EFM/ NST was likely to be associated with an increase in the incidence of CS without evidence of clear benefits.

Overall 100(39.68\%) women had meconium in liquor amnii, Wong [14] reported 20\%, 43 (17.06\%) women had FD with thick meconium, while $35(13.88 \%)$ had thin meconium in liquor with abnormalities, in IFHA or NST though, $11(4.36 \%)$ women with normal FHR also had thick meconium. Presence of meconium in liquor amnii, especially thick meconium, carries more risk of fetus being asphyxiated [12]. Also suspicious and pathologic tracings on EFM/NST were often found in the cases with meconium in liquor, though there was no significant difference in the Apgar scores (6.3 without meconium \& 7.15 with meconium). Use of IFHA in conjunction with EFM/NST could give better interpretation of foetal status.

Neilson [9] after Cochrane review advocated use of Foetal Electrocardiogram (ECG) for foetal monitoring during labour. His findings provided modest support to the use of fetal ST waveform analysis when a decision has been made to undertake continuous electronic FHR monitoring during labour.

Simple assessment techniques need to be widely available for diagnosis of non reassuring foetal status or FD. The clinician should reconsider the diagnosis made by FHR changes alone i.e. IFHA \& electronic foetal heart monitoring, and should interprete findings with some reservations due to limited accuracy in diagnosing foetal hypoxia, asphyxia and subsequent neonatal outcome.

The CS for FD with doubtful authenticity continue, due to defensive practices by clinicians worldwide, fear of litigation associated with poor labour outcome. More accurate techniques of foetal assessment, need to be available for wide use. So research must continue. Further research is also needed for non-conventional or unknown risk factors, which might be responsible for fetal asphyxia, there by leading to diagnosis of FD, also for establishing the authenticity of the diagnosis of non-reassuring fetal status, as well as appropriateness of the interventions done for the same.

\section{Acknowledgement}

We thank the patients for their consent and cooperation.

\section{References}

1. Parer JT, Livingston EG. What is fetal distress? Am J Obstet Gynecol. 1990; 162: $1421-1425$.

2. Committee on Obstetric Practice. ACOG Committee Opinion. Number 326. 2005. Inappropriate use of the terms fetal distress and birth asphyxia. Obstet Gynecol. 2005; 106: 1469-1470.

3. American College of Obstetricians and Gynecologists. ACOG Practice Bulletin No. 106: Intrapartum fetal heart rate monitoring: nomenclature, interpretation, and general management principles. Obstet Gynecol. 2009; 114: 192.

4. American Academy of Pediatrics, American College of Obstetricians and Gynecologists. Guidelines for perinatal care. $6^{\text {th }}$ ed. Elk Grove Village (IL): AAP; Washington, DC: ACOG. 2007; 103: 284-286

5. Al-Abid A. Foetal Distress. 2004

6. Odongo BE, Ndavi PM, Gachuno OW, Sequeira E. Cardiotocography and perinatal outcome in women with and without meconium stained liquor. East Afr Med J. 2010; 87: 199-204.

7. Low JA, Pickersgill H, Killen H, Derrick EJ. The prediction and prevention of intrapartum fetal asphyxia in term pregnancies. Am J Obstet Gynecol. 2001; 184: $724-730$.

8. Rosser J. Confidential Enquiry into Stillbirths and Deaths In Infancy (CESDI). Highlights of the $5^{\text {th }}$ annual report (Part II). Pract Midwife. 1998; 1: 22.

9. Nelson KB, Dambrosia JM, Ting TY, Grether JK. Uncertain value of electronic fetal monitoring in predicting cerebral palsy. N Engl J M. 1996; 334: 613-619.

10. Houston TP, Elster AB, Davis RM, Deitchman SD. The US Preventive Services Task Force Guide to Clinical Preventive Services, AMA Council on Scientific Affairs. AJPMs. 1998; 14: 374-376.

11. Macones GA, Hankins GD, Spong CY, Hauth J, Moore T. The 2008 National Institute of Child Health and Human Development workshop report on electronic fetal monitoring: update on definitions, interpretation, and research guidelines. Journal of Obstetric, Gynecologic, \& Neonatal Nursing. 2008; 37 : 510-515.

12. Chauhan SP, Magann EF, Scott JR, Scardo JA, Hendrix NW, Martin Jr JN. Cesarean delivery for fetal distress: rate and risk factors. Obstet Gynecol Surv. 2003; 58: 337-350.

13. Devane D, Lalor JG, Daly S, Mcguire W, Smith V. Cardiotocography versus intermittent auscultation of fetal heart on admission to labour ward for assessment of fetal wellbeing. Cochrane DatabaseSyst. 2012.

14. Wong SF, Chow KM, Ho LC. The relative risk of'fetal distress' in pregnancy associated with meconium-stained liquor at different gestation. J Obstet Gynaecol. 2002; 22: 594-599.
Austin J Obstet Gynecol - Volume 4 Issue 2 - 2017 Submit your Manuscript | www.austinpublishing group.com Chhabra et al. (C) All rights are reserved
Citation: Chhabra S and Mandar K. Concerns about Validity of Caesarian Section for Foetal Distress with No Risk in Mother - Baby. Austin J Obstet Gynecol. 2017; 4(2): 1072. 